City University of New York (CUNY)

CUNY Academic Works

2010

\title{
A Microfluidic Device to Establish Concentration Gradients Using Reagent Density Differences
}

Qingjun Kong

Richard A. Able, Jr.

Veronica Dudu

Maribel Vazquez

CUNY City College

\section{How does access to this work benefit you? Let us know!}

More information about this work at: https://academicworks.cuny.edu/cc_pubs/351

Discover additional works at: https://academicworks.cuny.edu

This work is made publicly available by the City University of New York (CUNY).

Contact: AcademicWorks@cuny.edu 


\section{A Microfluidic Device to Establish Concentration Gradients Using Reagent Density Differences}

\section{Richard A. Able, Jr.} Veronica Dudu Maribel Vazquez ${ }^{1}$ e-mail: vazquez@ccny.cuny.edu

Department of Biomedical Engineering, The City College of The City University of New York (CCNY),

Room 403D, Steinman Hall, 160 Convent Avenue, New York, NY 10031
Microfabrication has become widely utilized to generate controlled microenvironments that establish chemical concentration gradients for a variety of engineering and life science applications. To establish microfluidic flow, the majority of existing devices rely upon additional facilities, equipment, and excessive reagent supplies, which together limit device portability as well as constrain device usage to individuals trained in technological disciplines. The current work presents our laboratory-developed bridged $\mu$ Lane system, which is a stand-alone device that runs via conventional pipette loading and can operate for several days without need of external machinery or additional reagent volumes. The bridged $\mu$ Lane is a two-layer polydimethylsiloxane microfluidic device that is able to establish controlled chemical concentration gradients over time by relying solely upon differences in reagent densities. Fluorescently labeled Dextran was used to validate the design and operation of the bridged $\mu$ Lane by evaluating experimentally measured transport properties within the microsystem in conjunction with numerical simulations and established mathematical transport models. Results demonstrate how the bridged $\mu$ Lane system was used to generate spatial concentration gradients that resulted in an experimentally measured Dextran diffusivity of $(0.82 \pm 0.01)$ $\times 10^{-6} \mathrm{~cm}^{2} / \mathrm{s}$. [DOI: $10.1115 / 1.4002797$ ]

Keywords: microfluidic device, concentration gradients, Dextran

\section{Introduction}

Microfabrication techniques have been widely adapted to develop systems that generate specific spatial chemical concentrations and concentration gradient profiles in real time [1-6]. Microfluidics is often considered a central technology for miniaturized systems developed for chemical, biological, and medical applications due in large part to their proven ability to increase throughput and reduce experimental cost [5,7-9]. Various groups have developed microfluidic devices that generate linear [10-12] and nonlinear concentration gradients [13-16] via conventional forced fluid flow [17-21], as well as via diffusion across laminar streams [22,23], diffusion along interconnecting channels $[15,24]$, or in combination with electroporation [25] and numerous other methods [26,27].

While researchers have increasingly begun to combine multiple design features to achieve greater gradient control [28-34], the ultimate goal is often to manufacture devices that are easy to produce, minimize convective fluid flow, and maintain controlled gradients for extended periods of time [3,31,35-38]. One of the most common methods for generating steady-state gradients has been through free-diffusion of molecules between source and sink fluids separated by either a membrane or 3D gel $[4,34,35,39]$ or geometric obstructions $[40,41]$. These methods impede convective fluid flow, thereby enabling stable gradient formation over the life of the reagents. A comparable number of systems have achieved gradient control via microfluidic interfaces that are perpendicular to parallel flowing fluids $[33,42]$. Systems that generate such gradients directly $[7,32,43,44]$ or indirectly $[18]$ are able to maintain stable gradients for extended periods of time so long as desired input flow rates remain constant $[3,45]$.

\footnotetext{
${ }^{1}$ Corresponding author.

Contributed by the Bioengineering Division of ASME for publication in the JourNal of Biomechanical Engineering. Manuscript received March 25, 2010; final manuscript received September 17, 2010; accepted manuscript posted October 15, 2010; published online November 16, 2010. Assoc. Editor: Clark T. Hung.
}

A large drawback of many microfluidic designs is their dependence on multistage flow control and complex 3D transport phenomena in order to rapidly generate desired concentration gradients [46]. In addition, most microsystems continue to rely upon costly facilities and equipment for device fabrication, sample handling, and/or operation, including 3D photolithographic layering [12], piezoelectric microactuators [11], and external power supplies $[14,47,48]$. As a result, an additional microfluidic design goal has been to produce systems that eliminate the need for external parts while maintaining methods of generating gradients with distinctly different profiles [35]. Hence, there remains a bioengineering need for user-friendly systems that maintain given microenvironments via a combination of gradients and flow control in order to examine the numerous biological phenomena that occur across long time scales and confined spatial distances $[49,50]$.

The current study has developed a microfluidic device, called the bridged $\mu$ Lane system, which is a stand-alone device that operates continuously and autonomously without need of external equipment, power supplies, computerized apparatus, or clean room facilities. Instead, our system is fabricated in a conventional fume hood and sustains steady-state concentration gradients over a period of 2-3 days by exploiting the ultralow bulk velocities generated by density differences between the reagents used. In doing so, the bridged $\mu$ Lane can generate gradient profiles that span over five orders of magnitude over hour-long time scales that approach the microenvironments generated in vivo. In brief, the bridged $\mu$ Lane consists of two layers of the biocompatible material polydimethylsiloxane (PDMS). The top layer serves as a user interface to maintain consistent experimental conditions within large volume reservoirs that are fluidically connected to a closed bottom layer, where samples are transported and examined within a single microchannel. This work presents the design and fabrication of the bridged $\mu$ Lane system alongside experimental, numeri- 
$\mathbf{A}$

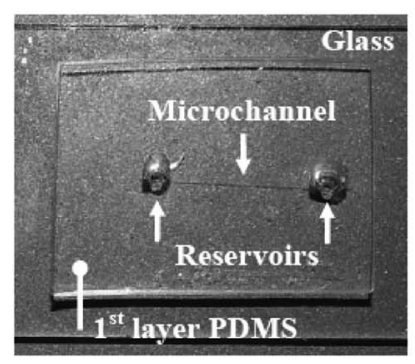

B

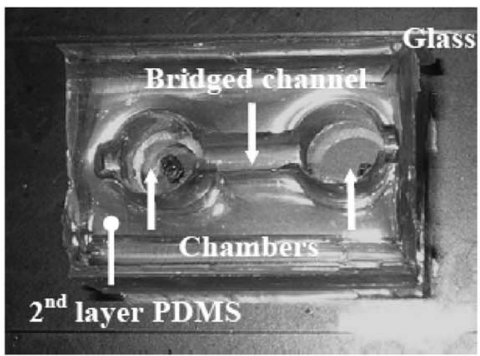

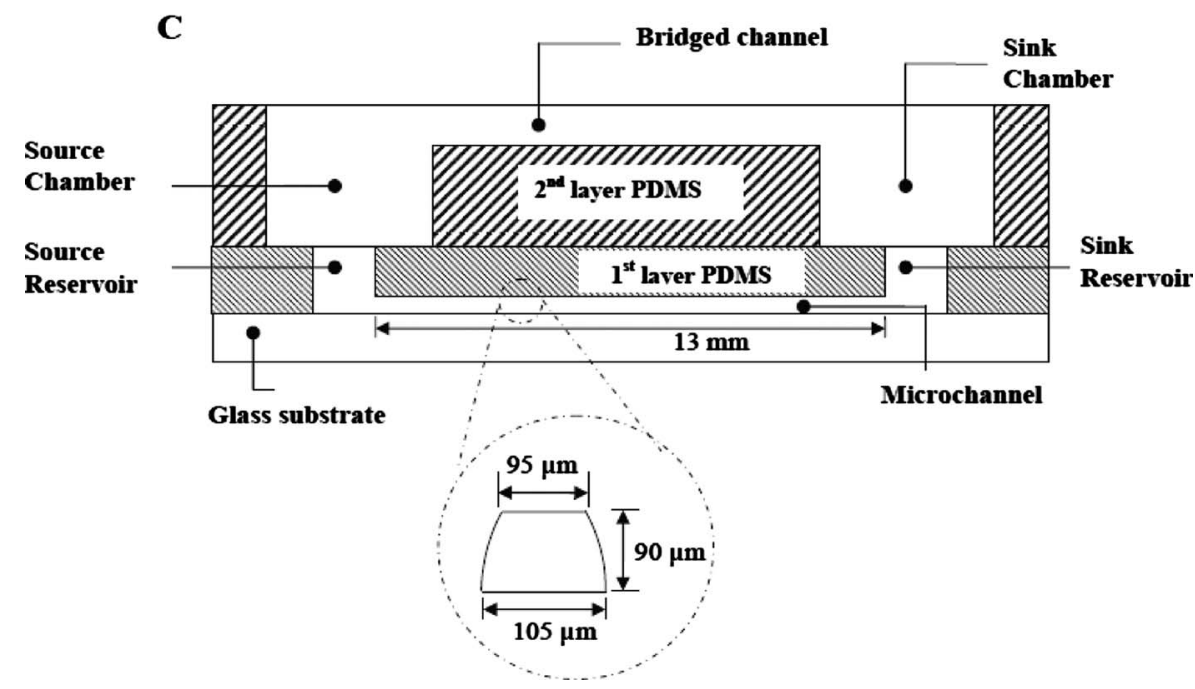

Fig. 1 Images and schematic of the bridged $\mu$ Lane system. After photolithography and elastomeric molding, a microchannel with two reservoirs was fabricated in polymerized PDMS, defined as the first layer PDMS of the bridged $\mu$ Lane system. (a) This PDMS layer was then bonded onto an ultrasonically cleaned microscope glass slide. (b) A second layer of PDMS, consisting of a bridge channel and two chambers, was molded on top of the first layer PDMS. This layer of PDMS was defined as the second layer PDMS, or user interface layer, of the system. (c) The chambers in the second layer PDMS were fluidically connected with the reservoirs in the first layer PDMS, as shown in the bridged $\mu$ Lane system schematic (not to scale). The microchannel approximately measures 13 $\mathrm{mm}$ in length, $90 \mu \mathrm{m}$ in depth, and $100 \mu \mathrm{m}$ in width (averaged with the upper side of $95 \mu \mathrm{m}$ and the lower side of $105 \mu \mathrm{m}$ ), as its semihemispherical cross section shown in inset.

cal, and mathematical validations of stable concentration gradients generated therein using fluorescently labeled Dextran as a test molecule.

\section{Materials and Methods}

2.1 Design. The bridged $\mu$ Lane system consists of two layers of PDMS with a closed microchannel and two fluidic reservoirs on the first layer and two larger fluidic chambers and an open bridge channel on the second or user interface layer. As shown in Fig. 1 $(a)$, the first layer contains two $9.0 \mu \mathrm{l}$ volume reservoirs called the source reservoir (SRR) and sink reservoir (SKR) connected by a single, closed microchannel, or $\mu$ Lane, of 13,000 $\mu \mathrm{m}$ length and $95 \mu \mathrm{m}$ hydraulic diameter using $\mathrm{D}_{\mathrm{h}}=4 \mathrm{~A} / \mathrm{P}$ [16] where $\mathrm{A}$ is the cross-sectional area and $\mathrm{P}$ is the perimeter. The system's second layer of PDMS is the user interface layer and consists of two cylindrical chambers, defined as the source chamber (SRC) and sink chamber (SKC), and a semicircular, open channel, known as the bridge channel (Fig. 1(b)). The SRC and SKC have $6 \mathrm{~mm}$ diameter and $6 \mathrm{~mm}$ height each $(170 \mu \mathrm{l})$ and are vertically and fluidically connected with the SRR and SKR in the first layer of the system, respectively. In addition, the SRC and SKC are connected to each other via the open bridge channel with $9 \mathrm{~mm}$ length and $2 \mathrm{~mm}$ depth. The overall system works by using the large volume chambers and bridge channel on the second layer to generate concentration gradients within the smaller volume microchannel on the first layer. Solution is manually loaded into the SRC until it has filled the SRR, microchannel, SKR, SKC, and bridge channel. Sample is then loaded into the SRC, dropwise and slowly, until the sample makes contact with the solution within the bridge channel to initiate system operation. The volume of each chamber $(170 \mu \mathrm{l})$ is designed to be much larger than the volume within each reservoir $(9 \mu \mathrm{l})$ and $\mu$ Lane $(0.1 \mu \mathrm{l})$ in order to facilitate manual loading via conventional pipette or syringe to initiate gradual transport into the microchannel with minimal channel entrance effects. The large ratio of chamber volume $(170 \mu \mathrm{l})$ to $\mu$ Lane volume $(0.1 \mu \mathrm{l})$ facilitates modeling of constant reagent concentration in the chambers during transport analysis. Further, the ratio of $\mu$ Lane length to diameter is substantially large $\left(\mathrm{L} / \mathrm{D}_{\mathrm{h}}=137\right)$ so as to enable computational modeling of one-dimensional transport within the microchannel. Lastly, the ratio of each reservoir volume $(9 \mu \mathrm{l})$ to the $\mu$ Lane volume 
$\left(\begin{array}{ll}0.1 & \mu l\end{array}\right)$ is also large $\left(\mathrm{V}_{\mathrm{SRR}} / \mathrm{V}_{\mu \text { Lane }}=27\right)$ in order to sustain continuous transport between each reservoir and the microchannel over long experimental time scales.

Without the bridge channel, the $\mu$ Lane channel readily facilitates transport via convection as even small differences in the liquid levels of the SRR and SKR will generate hydrostatic pressure gradients known to cause microfluidic flow $[5,6,11]$. The advantage of the so-called bridged $\mu$ Lane system is that the bridge channel is used to balance solution volumes within the SRC and $\mathrm{SKC}$ in order to eliminate hydrostatic pressure differences between the chambers. As such, the addition of sample and/or reagent with a different density into the SRC on the second layer then provides the driving force to generate a small microfluidic flow within the $\mu$ Lane channel in the first layer, which facilitates transport via convective diffusion. The large differences in volume ratios between chambers, reservoirs, and channels on the first and second layers of the device provide the dual advantage of enabling the modeling of transport processes as one-dimensional in the microchannel, as well as generating fluidic processes with very different time scales for achieving steady-state transport.

2.2 Manufacturing. System fabrication was performed via three different conventional manufacturing processes: (i) fabrication of microchannel-patterned mold using proximity photolithography, (ii) fabrication of the first layer PDMS using elastomeric molding and bonding, and (iii) fabrication of the second layer PDMS using elastomeric molding.

2.2.1 Photolithography. A precleaned glass substrate was coated with an adhesion promoter, Surpass 3000 (DisChem Inc., Ridgway, PA), and a $100 \mu \mathrm{m}$ thick layer of negative photoresist, SU-8 2075 (MicroChem Inc., Newton, MA), using a spin coating apparatus (Laurell Tech. Corp., North Wales, PA). The substrate was baked on a hot plate and irradiated with UV light using a laboratory-fabricated photomask containing desired microchannel patterns (intensity: $21,700-8900 \mu \mathrm{W} / \mathrm{cm}^{2}$ at $2-10$ in., Model B-100AP, UVP Inc., Upland, CA). Substrates were then rebaked, allowed to cool at room temperature $25^{\circ} \mathrm{C}$, and immersed in SU-8 developer (MicroChem Inc., Newton, MA) until photoresist patterns were visible. The patterned substrate exhibited dimensions of $13.0 \pm 0.1 \mathrm{~mm}$ length (mean \pm standard deviation $(\mathrm{SD})$ ) by $100 \pm 5 \mu \mathrm{m}$ width by $90 \pm 1 \mu \mathrm{m}$ height as measured via surface profilometer (Model SJ-301, Mitutoyo America Corp., Middlesex, NJ) and optical microscope (Nikon TE2000, Morrell Instrument Co. Inc., Melville, NY).

2.2.2 Fabrication of the First Layer PDMS. Liquid-state PDMS mixed with silicone elastomer curing agent (Dow Corning Corp., Midland, MI) was degassed and poured onto the photoresist-patterned substrate described above. After polymerization, the PDMS elastomer was peeled from the patterned substrates, resulting in open microchannels of $13.0 \pm 0.1 \mathrm{~mm}$ length by $100 \pm 5 \mu \mathrm{m}$-width and $90 \pm 1 \mu \mathrm{m}$ depth. Subsequently, it was punctured with $2 \mathrm{~mm}$ diameter pipette tips (Model 9 in. Pasteur pipette, Fisher Scientific Co., Agawam, MA) on both ends in order to generate two reservoirs with approximately $9 \mu \mathrm{l}$ volume each, defined as the SRR and SKR. The PDMS elastomer was bonded onto a precleaned glass substrate (unpatterned) by applying ozone generated via a high-frequency generator (Electro-Technic Products, Inc., Chicago, IL) onto both the PDMS and glass surfaces for $30 \mathrm{~s}$ each. The first layer PDMS was then generated, as shown in Fig. 1(a). Cross section uniformity of the closed microchannel itself within this first layer was verified using an optical microscope (Nikon TE2000). Since large machines, such as deep reactive-ion etching (DRIE) apparatus, were not used in the microfabrication, the cross section was semihemispherical [51-54] with a hydraulic diameter of $\mathrm{D}_{\mathrm{h}}=95 \mu \mathrm{m}$ as examined via optical microscope and profilometer, as shown in Fig. 1(c). This profile is of note because high aspect ratio, rectangular channels are extremely difficult to manufacture via this protocol [55].
2.2.3 Fabrication of the Second Layer PDMS. For the second layer of PDMS, or user interface layer, a laboratory-made acrylic mold was manufactured via milling machine to match the larger dimensions of the SRC, SKC, and bridge. After the acrylic mold was aligned on top of the first layer PDMS, the mixed solution of liquid-state PDMS and curing agent were poured around the acrylic mold to form a $6 \mathrm{~mm}$ thick second layer PDMS. After the PDMS was polymerized, the acrylic mold was removed. The second layer PDMS consisted of two cylindrical chambers and a semicircular, open channel or so-called bridge channel (Fig. 1(b)). The two chambers, with $6 \mathrm{~mm}$ diameter and $6 \mathrm{~mm}$ height each, were defined as the SRC and SKC. The SRC and SKC were vertically and fluidically connected with the SRR and SKR, respectively, in the first layer (Fig. 1(c)).

The complete bridged $\mu$ Lane system is thus composed of an open bridge channel that connects the open SRC and SKC on the second or user interface layer, as well as a closed microchannel that connects the SRR and SKR on the first layer.

2.3 Mathematical Modeling. Two-dimensional numerical simulations of the bridged $\mu$ Lane system were performed in order to model the transport within entire the microsystem, i.e., the microchannel, both the SRR and SKR in the first layer PDMS, the bridge channel, and both the SRC and SKC in the second layer PDMS. The mass transport within the entire microsystem was modeled using the 2D continuity equation (Eq. (1)), convective diffusion equation (Eq. (2)), momentum equation (Eq. (3)), and hydrostatic equation (Eq. (4)), as shown below [56],

$$
\begin{gathered}
\text { continuity equation: } \frac{\partial \rho}{\partial t}+\underline{\nabla}(\rho \cdot \underline{u})=0 \\
\text { convective-diffusion equation: } \frac{\partial C}{\partial t}+\underline{u} \cdot \underline{\nabla} C=D \nabla^{2} C \\
\text { momentum equation: } \rho\left(\frac{\partial \underline{u}}{\partial t}+\underline{u} \cdot \underline{\nabla} \underline{u}\right)=-\underline{\nabla} p+\mu \nabla^{2} \underline{u} \\
\text { hydrostatic equation:- } \underline{\nabla} p+\rho \underline{g} h=0
\end{gathered}
$$

where $\rho\left(\mathrm{kg} / \mathrm{m}^{3}\right)$ is reagent density, $\underline{u}(\mathrm{~m} / \mathrm{s})$ is fluid velocity, y $(\mathrm{m})$ is the vertical direction, $\mathrm{x}(\mathrm{m})$ is the horizontal direction, $C(\mathrm{~g} / \mathrm{l})$ is solution concentration, $D\left(\mathrm{~m}^{2} / \mathrm{s}\right)$ is the diffusion coefficient, or diffusivity, of the reagent molecule, $\mathrm{p}(\mathrm{Pa})$ is hydrodynamic pressure, and $\mathrm{h}(\mathrm{m})$ is the height of the first and second layer PDMS $(\mathrm{m})$. These coupled equations were solved using finite element methods. Note that all physical boundaries of the microsystem were regarded as insulated boundaries of mass transfer and momentum transport because only trace amounts of Dextran were observed to permeate through PDMS wall boundaries during experimental time scales of up to 7 days. Transport within PDMS across 1-2 days is therefore neglected in this analysis [57]. For ease of explanation, mathematical modeling will be described within four separate control regions: (i) the SRC in the second layer and the SRR in the first layer, (ii) the microchannel in the first layer, (iii) the SKR in the first layer and the SKC in the second layer, and (iv) the bridge channel in the second layer. Finite-element-analysis (FEA) software FEMLAB version 3.4 (Comsol Inc., Burlington, MA) was used to solve these four coupled equations simultaneously within the four interconnected regions described above. A fine mesh of 1504 elements was used, which confined the distance between nodes to approximately $8 \mu \mathrm{m}$ and a computational time of less than $3 \mathrm{~min}$. Note that simulation results remained approximately unchanged $(<1 \%)$ when finer meshes were used.

2.3.1 SRC to $S R R$. The initial reagent concentration within the SRR was set to $0 \mu \mathrm{g} / \mathrm{ml}$ because no sample reagent (only buffer or deionized water) was directly added into the reservoir, while the initial molecule concentration within the SRC was set to 
$40 \mu \mathrm{g} / \mathrm{ml}$ to reflect the sample reagent concentration used during experiments. The concentration within the SRC was held constant throughout simulations because the large chamber to reservoir volume ratio (140:1) maintained an approximately constant reagent concentration during experiments. As a result, the molecule concentration at the interface between the SRC and the SRR was fixed at $\mathrm{C}=40 \mu \mathrm{g} / \mathrm{ml}$. The reagent concentration at the interface between the SRR and the entrance of the microchannel $\left(\mathrm{C}_{\mathrm{x}=0-}\right)$ was modeled as equal to the reagent concentration at the interface within the microchannel $\left(\mathrm{C}_{\mathrm{x}=0+}\right)$, i.e., $\mathrm{C}_{\mathrm{x}=0-}=\mathrm{C}_{\mathrm{x}=0+}$, indicative of a continuous boundary.

The initial velocity entering from the SRC and within the SRR was assumed to be zero. Lastly, the boundary condition of velocity at the interface between the SRR and the microchannel was set $\mathrm{V}_{\mathrm{x} \mid \mathrm{x}=0-}=\mathrm{V}_{\mathrm{x} \mid \mathrm{x}=0+}$, as a continuous boundary. The hydrodynamic pressures within the SRC and SRR were calculated using the hydrostatic equation (Eq. (4)).

2.3.2 Microchannel. The transport within the microchannel from the SRR to the SKR was modeled as one-dimensional because of the large ratio between the microchannel length and its hydraulic diameter (137:1). The initial concentration of reagent within the entire microchannel was set to $\mathrm{C}=0 \mu \mathrm{g} / \mathrm{ml}$ as no reagent was applied in the beginning of experiments. The boundary at the entrance of the microchannel was set as continuous boundary $\left(\mathrm{C}_{\mathrm{x}=0-}=\mathrm{C}_{\mathrm{x}=0+}\right)$ as mentioned. Similarly, the boundary at the exit of the microchannel $(\mathrm{x}=\mathrm{L})$ was also set as a continuous boundary, i.e., $\mathrm{C}_{\mathrm{x}=\mathrm{L}-}=\mathrm{C}_{\mathrm{x}=\mathrm{L}+}$, as the concentration within the SKR at the interface between the microchannel and the reservoir $\left(\mathrm{C}_{\mathrm{x}=\mathrm{L}-}\right)$ was regarded to be equal to the concentration within the SKR at the interface $\left(\mathrm{C}_{\mathrm{x}=\mathrm{L}+}\right)$. The initial velocity within the microchannel was zero, while the boundary conditions at the entrance of the microchannel and the exit of the microchannel were regarded as continuous boundaries, i.e., $\mathrm{V}_{\mathrm{x} \mid \mathrm{x}=0-}=\mathrm{V}_{\mathrm{x} \mid \mathrm{x}=0+}$ and $\mathrm{V}_{\mathrm{x} \mid \mathrm{x}=\mathrm{L}-}=\mathrm{V}_{\mathrm{x} \mid \mathrm{x}=\mathrm{L}+}$.

2.3.3 SKR to SKC. The initial reagent concentration within the SKR and $\mathrm{SKC}$ was set to $\mathrm{C}=0 \mu \mathrm{g} / \mathrm{ml}$ as no reagent was added at the beginning of experiments. The concentration at the boundary between the SKC and the SKR was set to $\mathrm{C}=0 \mu \mathrm{g} / \mathrm{ml}$ and the boundary between the SKR and the microchannel $(\mathrm{x}=\mathrm{L})$ was regarded as a continuous boundary, i.e., $\mathrm{C}_{\mathrm{x}=\mathrm{L}-}=\mathrm{C}_{\mathrm{x}=\mathrm{L}+}$. The velocity within the SKR was initially zero. The velocity at the interface between the SKC and the SKR was set to be zero, while the boundary condition of velocity at the interface between the microchannel and the SKR was set as continuous boundary, i.e., $\mathrm{V}_{\mathrm{x} \mid \mathrm{x}=\mathrm{L}-}=\mathrm{V}_{\mathrm{x} \mid \mathrm{x}=\mathrm{L}+}$. The pressure in the SKR was calculated via hydrostatics.

2.3.4 Bridge Channel. The initial concentrations of the SRC and the SKC were set to $40 \mu \mathrm{g} / \mathrm{ml}$ and $0 \mu \mathrm{g} / \mathrm{ml}$, respectively, and maintained constant during simulations. The initial reagent concentration within the bridge channel was also $0 \mu \mathrm{g} / \mathrm{ml}$ as no reagent was added. The concentrations at the boundaries of the interface between the SRC and the bridge channel and the interface between the SKC and the bridge channel were also set to $40 \mu \mathrm{g} / \mathrm{ml}$ and $0 \mu \mathrm{g} / \mathrm{ml}$, respectively.

2.4 Experimental Measurement. Fluorescently labeled Dextran solutions (Cat. No. D22910, 10 kDa, molecular probes, Eugene, OR) and $1.9 \mu \mathrm{m}$ diameter fluorescent beads (Duke Scientific, Cat. No. G0200, Palo Alto, CA) were added into the system in order to explicitly measure Dextran transport within the bridged $\mu$ Lane system. First, phosphate buffer saline (PBS) solution (Mediatech Inc., Herndon, VA) containing the $1.9 \mu \mathrm{m}$ diameter fluorescent beads with a concentration of $10^{4}$ beads/ml was injected into the microchannel using a $1 \mathrm{ml}$ syringe (Becton, Dickinson and Co., Franklin Lakes, NJ). These beads were used to optically measure bulk flow velocity within the reservoirs, microchannel, and chambers of the system. The PBS solution was then pipetted into the SKC and the bridge. Note that the PBS solution was added dropwise and slowly into the bridge and the SKC so as to prevent dilution of the fluorescent source. Afterward, solutions of Dextran at a concentration of $40 \mu \mathrm{g} / \mathrm{ml}$ were added dropwise into the SRC only until it contacted the PBS solution present in the bridge. After the contact was made, solutions of fluorescent beads and solutions of Dextran were observed to migrate significantly more slowly within the microchannel from the SRR to the SKR. The systems were then placed in a fluorescent microscope for fluorescent intensity measurement. The fluorescent detection positions within the microchannel were set at $3 \mathrm{~mm}, 5 \mathrm{~mm}, 8 \mathrm{~mm}$, and $11 \mathrm{~mm}$ away from the entrance of the microchannel.

2.5 Microscope Imaging and Fluorescent Measurement. An inverted epimicroscope (Nikon TE2000) with a $20 \times$ microscope objective (Nikon Plant 20×, Morrell Instrument Co. Inc., Melville, NY) was used to image the $\mu$ Lane systems via a cooled charge-coupled device (CCD) camera (CoolSNAP EZ, Photometrics, Tucson, AZ) with NIKON software (Nikon Instrument Element 2.30 with $6 \mathrm{D}$ module, Morrell Instrument Co. Inc., Melville, NY). A shutter (HF204, Prior Scientific Inc., Rockland, MA) mounted in the Nikon TE2000 was used to control the exposure time.

An excitation light with a wavelength of $488 \mathrm{~nm}$ was applied onto the channel during Dextran transport so that the emitted fluorescence was collected via microscope objective. The intensity values of fluorescence collected at detection positions $5 \mathrm{~mm}, 8$ $\mathrm{mm}$, and $11 \mathrm{~mm}$ away from the SRR were plotted over time. Meanwhile, the movement of fluorescent beads within the microchannel was recorded in order to measure the mean bulk flow velocity. Experiments were repeated four times. In each repeated experiment, a new bridged $\mu$ Lane system was used to measure the fluorescent intensity and bulk flow velocity at different detection positions over time. The results were processed using the Student's t-test and presented in the format of average \pm standard deviation.

2.6 Data Processing and Plotting. The experimental data of fluorescent intensity ( 8 bit values between 0 and 256) as a function of time and the simulation data as a function of time were normalized to values between 0 and 1 and plotted in the same diagram using software (ORIGIN 7.5, Origin Lab Corp., Northampton, MA). Note that some dimensionless normalized experimental data slightly exceeded 1 because of experimental noise. The normalized experimental data were fitted into a curve $\left(\mathrm{R}^{2} \geq 99.8 \%\right)$ using the Hill function, a category of Sigmoidal functions provided by the software, in order to better compare with the simulated data. The Hill function is given as

$$
y=V_{\max } \cdot \frac{x^{n}}{k^{n}+x^{n}}
$$

where $V_{\max }$ is the asymptotic value of the experimental data, $k$ and $n$ are empirical constants given by the software, and $x$ and $y$ are axis coordinates. The residual difference, defined as the absolute difference between each individual data point from the Hillfunction fit curve and each individual data point from the simulation curves at the same corresponding time point, was analyzed using the Student's t-test in order to identify the simulation curve that was closest to the Hill-function fit curve of the experimental data. The simulation curve with the least standard deviation of residual difference $(<2 \%)$ and the least average residual difference at all the data points $(<1 \%)$ was considered to be characteristic of the diffusion coefficients in free solution.

\section{Results}

The bridged $\mu$ Lane system was designed to generate steadystate concentration gradients within microchannels via reagent concentration difference without relying upon external equipment, power, or reagent volumes. A fluorescent reagent, mathematical modeling, and numerical simulations were used to examine the 
$\mathbf{A}$

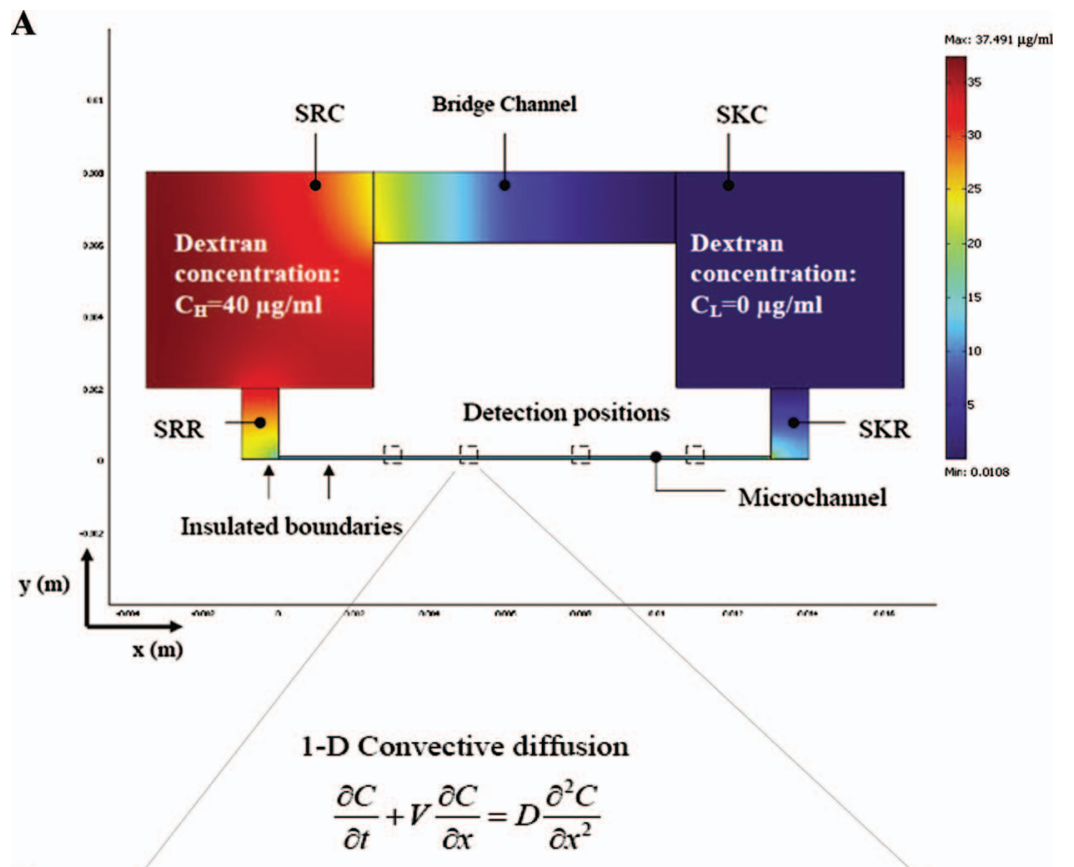

B

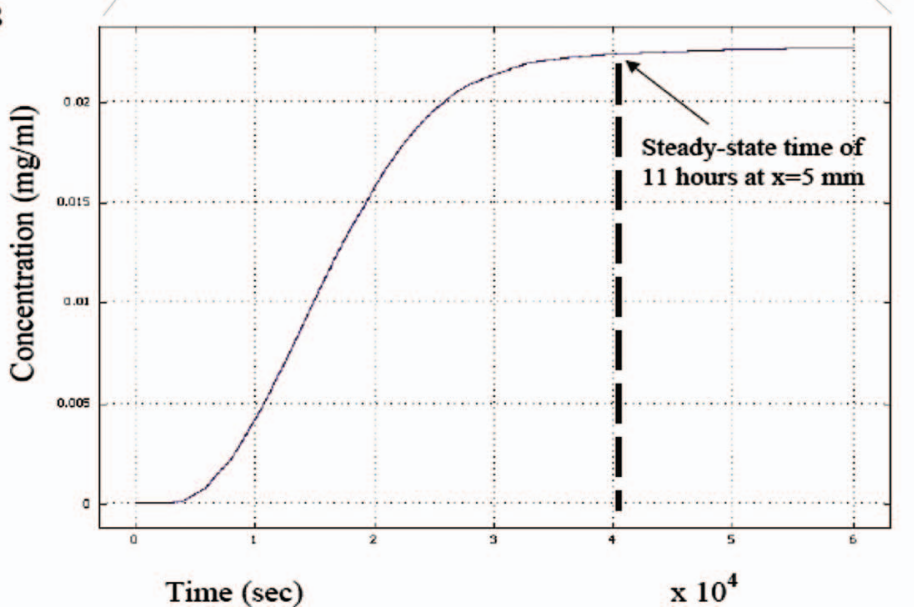

Fig. 2 Simulation of Dextran transport within the bridged $\mu$ Lane system. (a) The SRC, SRR, microchannel, SKR, SKC, and bridge channel were modeled using finite element software. The Dextran concentration in the SRC was set to a maximum of $C_{H}=40 \mathrm{ng} / \mathrm{ml}$, while the Dextran concentration in the SKC was set to a minimum of $C_{L}=0 \mathrm{ng} / \mathrm{ml}$. The bulk velocity within the microchannel was measured using fluorescent beads and inputted into the Dextran transport model for numerical simulation. All boundaries of the bridged $\mu$ Lane system were modeled as insulated from mass transfer. Dextran transport at different positions within the microchannel, $x=5 \mathrm{~mm}, x=8 \mathrm{~mm}$, and $x=11 \mathrm{~mm}$, was examined over time. One-dimensional convective diffusion equation was used to model the transport of Dextran within the microchannel, where $\mathrm{C}$ represents concentration, $\mathrm{V}$ is bulk velocity, and $\mathrm{D}$ is the diffusion coefficient. (b) The simulation illustrates that the Dextran concentration at a representative microchannel position of $x=5 \mathrm{~mm}$ increases over time and reaches steady-state in $11 \mathrm{~h}$.

concentration gradients generated within the microsystem. Results compared experimentally measured values of bulk velocity, diffusivity, and concentration to those predicted mathematically and derived numerically.

3.1 Mathematical Predictions. FEMLAB simulations generated concentration distributions within the bridged $\mu$ Lane system, as illustrated in Fig. 2. As seen, SRC and SKC concentrations remained approximately constant, while significant reagent concentration distributions were seen within the SRR and SKR.
Importantly, finite element method (FEM) analysis predicted that the reagent concentration varied by two orders of magnitude along the microchannel length. The time response of Dextran via FEM analysis shown in Fig. 2(b) illustrates how the reagent concentration reaches steady-state within $11 \mathrm{~h}$ midchannel at approximately $5 \mathrm{~mm}$. Bulk flow present in the microchannel was examined via Reynolds number (Re) and Peclet number $(\mathrm{Pe})$ to analyze its fluidic properties. The Reynolds number $(\mathrm{Re})$ is a dimensionless 

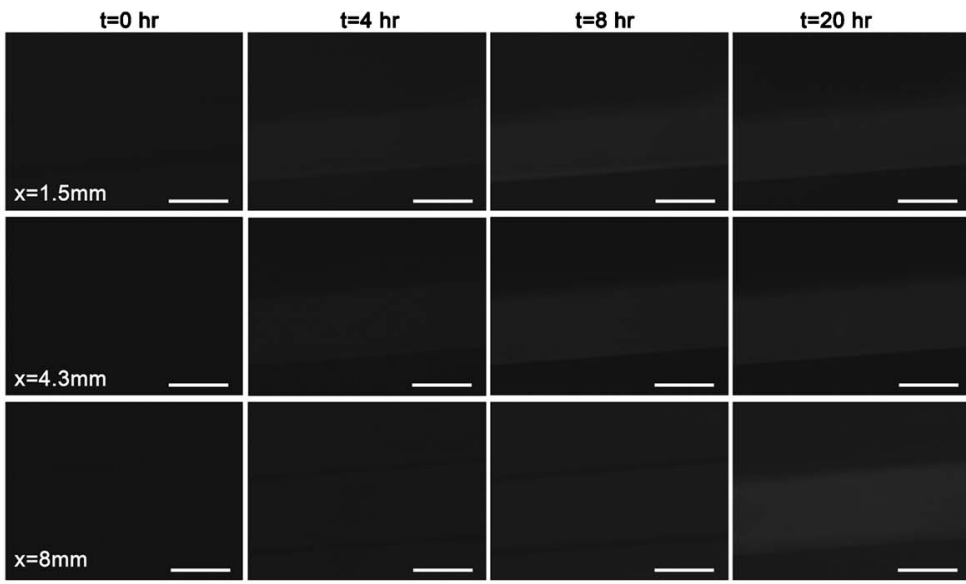

Fig. 3 (a) Images of Dextran transport within the microchannel at three representative positions over time. Fluorescence was initially observed throughout the microchannel in approximately $4 \mathrm{~h}$ with increasingly fluorescence intensity measured for up to $20 \mathrm{~h}$. (b) Average intensity values measured every hour across three different channel sections are plotted to illustrate the Dextran concentration profile obtained experimental at steady-state. Scale: $100 \mu \mathrm{m}$.

number that measures the ratio of inertial forces to viscous forces, defined as [56]

$$
\operatorname{Re}=\rho \mathrm{Vd} / \mu
$$

where $\rho$ is fluid density $\left(\mathrm{kg} / \mathrm{m}^{3}\right), \mathrm{V}$ is flow velocity $(\mathrm{m} / \mathrm{s}), \mathrm{d}$ is characteristic length $(\mathrm{m})$, and $\mu$ is fluid viscosity (Pa s). This value was predicted to be less than $10^{-3}$ in our model, indicative of viscous, laminar flow within the microchannel, with a bulk velocity of $0.17 \mu \mathrm{m} / \mathrm{s}$. The Peclet number $(\mathrm{Pe})$ is a dimensional number that measures the ratio of convective bulk flow to diffusion, defined as [56]

$$
\mathrm{Pe}=\mathrm{VL} / \mathrm{D}
$$

where $\mathrm{V}$ is fluid velocity $(\mathrm{m} / \mathrm{s}), \mathrm{L}$ is the length of channel $(\mathrm{m})$, and $\mathrm{D}$ is the diffusion coefficient $\left(\mathrm{m} / \mathrm{s}^{2}\right)$. Using the diffusion coefficient of Dextran reported in the literature to range from 0.8 $\times 10^{-6} \mathrm{~cm}^{2} / \mathrm{s}$ to $0.84 \times 10^{-6} \mathrm{~cm}^{2} / \mathrm{s}$ [58-62] and the average bulk flow velocity estimated from the mathematical models, the Pe was predicted to be between 16 and 25. Such values illustrate that bulk flow dominated the Dextran transport within the microchannel even at the low Re and velocities predicted mathematically via the Poiseuille flow model $[16,56]$.

Figure 2 also illustrates concentration differences within the bridge channel near the SRC after $23 \mathrm{~h}$. However, the volume of the bridge is over $10^{4}$ times larger than the volume of the microchannel, such that there is no backflow as a result of microchannel transport. In addition, concentration gradients within the bridge are diffusion-dominated, and as such the bridge does not equilibrate concentrations within the SRC and SKC within experimental time scales of 5-7 days given the large chamber volumes and absence of measurable bulk flow. FEMLAB analysis indicates that reagent equilibration within the second PDMS layer requires upward of $200 \mathrm{~h}$ given the system volumetric dimensions.

3.2 Experimental Measurement of Transport. Experimental measurements of the fluorescent intensity of Dextran samples and fluidic flow of fluorescent microbeads within the bridged $\mu$ Lane were performed in order to verify the transport parameters predicted by our model. No significant change of fluorescent intensity $(<1 \%)$ was measured within the SRC and SKC, indicative of approximately constant concentrations during experiments, i.e., $40 \mu \mathrm{g} / \mathrm{ml}$ and $0 \mu \mathrm{g} / \mathrm{ml}$, respectively. Transport of solutions of fluorescent Dextran and fluorescent beads was imaged within the system and observed to displace along the SRR, microchannel, and SKR with increasing fluorescent intensity by time, as shown in Figs. 3 and 4, respectively. As can be seen, Dextran fluorescence intensity was measured at different channel positions over a $20 \mathrm{~h}$ period and plotted to illustrate the time response within the bridged $\mu$ Lane system. The experimental data shown are within $9 \%$ of the response predicted via FEM simulation, indicating that a steady-state Dextran concentration is experimentally reached midchannel after approximately $12 \mathrm{~h}$ (as per Fig. 3 ).

In addition, bulk flow within the system was examined using fluorescent beads with and without the use of the bridge channel. As shown in Fig. 4, beads were seen to migrate with different velocities in the microsystem with and without the bridge channel. An average bulk velocity of $\mathrm{V}_{\text {avg }}=92.3 \pm 1.4 \mu \mathrm{m} / \mathrm{s}$ was measured within the $\mu$ Lane system in the absence of the bridge channel $(n=12)$, but an average velocity of $V_{a v g}=0.37 \pm 0.11 \mu \mathrm{m} / \mathrm{s}$ was measured within the bridged $\mu$ Lane system $(n=16)$. In addition, no bulk flow was measured within the bridge channel when beads were used, confirming that Dextran transport within the
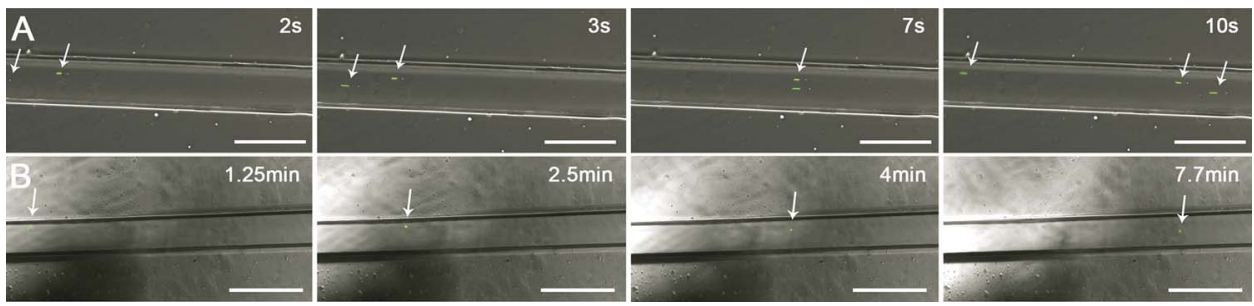

Fig. 4 Images of the bulk flow of beads within a $\mu$ Lane device (a) in the absence of the bridge channel and $(b)$ within the bridged $\mu$ Lane system. Images illustrate the motion of individual beads across a fixed, representative channel section. Scale: $100 \mu \mathrm{m}$. 
bridge was diffusion-dominated and did not significantly affect the transport of Dextran within the microchannel nor alter concentrations of the large volume SRC and SKC over experimental time scales.

As a validation experiment, the diffusion coefficient of Dextran was measured using the bridged $\mu$ Lane system by comparing the transport imaged at four different detection positions within the microchannel, $x=3 \mathrm{~mm}, \mathrm{x}=5 \mathrm{~mm}, \mathrm{x}=8 \mathrm{~mm}$, and $\mathrm{x}=11 \mathrm{~mm}$. The fluorescent intensity of Dextran measured experimentally over $96 \mathrm{~h}$ at these four specific detection positions was normalized and shown in Fig. 5. In addition, normalized FEM computermodeled predictions of Dextran concentration at the corresponding detection positions were computed and plotted alongside the experimental data in this figure. In the simulations, numerical predictions utilized Dextran diffusivity values between $D=1.0$ $\times 10^{-6} \mathrm{~cm}^{2} / \mathrm{s}$ and $D=0.7 \times 10^{-6} \mathrm{~cm}^{2} / \mathrm{s}$ and a measured bulk velocity of $\mathrm{V}_{x}=0.37 \mu \mathrm{m} / \mathrm{s}$ for a wide range of examinations. Computer-generated predictions from our numerical model were within $6 \%$ of each experimentally measured intensity. Using these corroborating results, we illustrate that Dextran transport within the bridged $\mu$ Lane system occurred with a diffusivity of $(0.82 \pm 0.01) \times 10^{-6} \mathrm{~cm}^{2} / \mathrm{s}$. This value is in line with the measured diffusivity of Dextran in vitro and in vivo reported to be between $0.80 \times 10^{-6} \mathrm{~cm}^{2} / \mathrm{s}$ and $0.84 \times 10^{-6} \mathrm{~cm}^{2} / \mathrm{s}$ [58-62].

Using the bridged $\mu$ Lane-derived diffusivity of Dextran and the average bulk velocity, the time to reach steady-state for the entire microchannel length of $\mathrm{L}=13 \mathrm{~mm}$ was computed to be 40 $h$. In addition, the concentration gradient profile $G$ within the system microchannel at steady-state spanned five orders of magnitude with minimal, shallow gradients of $\mathrm{G}=10^{-3} \mathrm{ng} /(\mathrm{ml} \mathrm{mm})$ near the SRR and larger, highly nonlinear gradients of $G$ $=10^{1} \mathrm{ng} /(\mathrm{ml} \mathrm{mm})$ near the SKR.

\section{Discussion}

The ability to produce mathematically validated chemical concentrations and concentration gradients is essential for the study of micro- and nanoscale transport within microfluidic devices. The bridged $\mu$ Lane system generates spatially distributed concentration gradients within microenvironments over time by exploiting the density difference between the reagents used. Although these differences are minimal, they are the driving forces that generate pressure differences between the SRR and SKR to result in bulk flow along the connecting microchannel. Importantly, the bridge channel was incorporated into the system in order to diminish bulk flow in the microchannel to ultralow levels that enabled autonomous operation for several days without need of replenished reagent volumes, external pumps, or power supplies.

The bridged $\mu$ Lane system utilizes ultralow bulk flow as an advantage as convective transport rapidly decreases the time required for reagent concentration to reach steady-state along the microchannel length. In the absence of the second layer PDMS for hydrostatic balance, for example, experiments illustrated that a submicroliter volume difference generated bulk flows within the microchannel on the order of $92.3 \mu \mathrm{m} / \mathrm{s}$, which would rapidly deplete the system within $1 \mathrm{~h}$. In the absence of bulk flow, however, Dextran transport via diffusion alone (using the average of cited Dextran diffusivities, $0.82 \times 10^{-6} \mathrm{~cm}^{2} / \mathrm{s}$ ) would require over $470 \mathrm{~h}$ to reach a steady-state gradient within a $13 \mathrm{~mm}$ long microchannel instead of the $40 \mathrm{~h}$ reported here. These minute bulk flows helped generate concentration gradients that spanned five orders of magnitude within the same experimental device. Larger bulk flows would have generated smaller differences in concentration gradients, perhaps requiring multiple systems to examine a range of gradients, as well as required additional reagent volumes and/or pumping per experiment.

Lastly, while other devices rely upon machined parts and complex 3D assembly, our system accomplishes microfluidic transport via inexpensive PDMS layering to accent the device's ease of use.
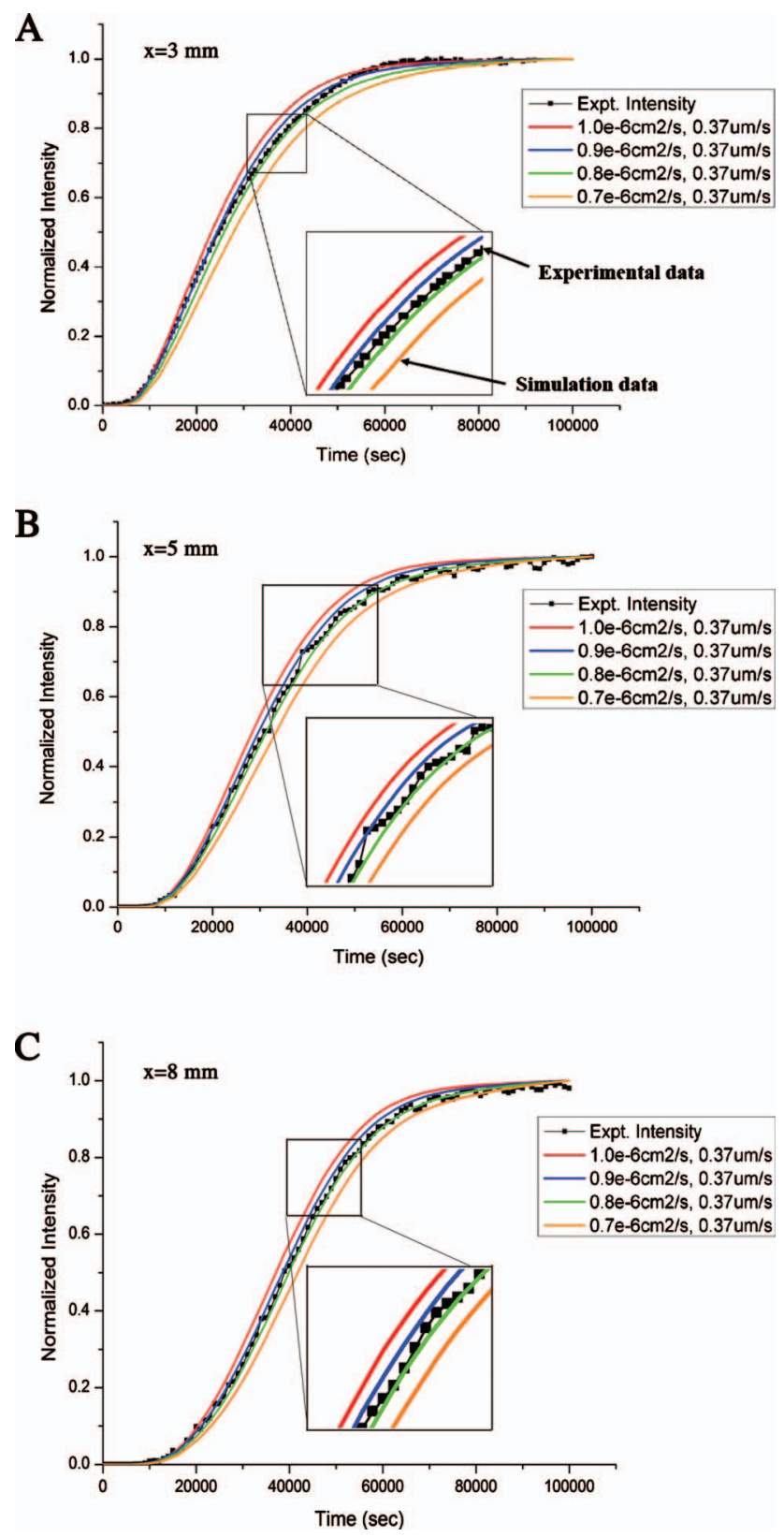

Fig. 5 Graph of normalized experimental data obtained from Dextran transport experiments at detection positions within the bridged $\mu$ Lane system, compared against normalized simulation data generated from the 1D convective diffusion equation. These normalized data were plotted as a function of time. A range of Dextran diffusion coefficients between 0.7 $\times 10^{-6} \mathrm{~cm}^{2} / \mathrm{s}$ and $1.0 \times 10^{-6} \mathrm{~cm}^{2} / \mathrm{s}$ and the experimentally measured mean bulk velocity for Dextran of $V=0.37 \mu \mathrm{m} / \mathrm{s}$ were used in the numerical simulation to generate the Dextran concentration profiles shown at different detection positions over time.

Fabrication does not require clean room facilities with conventional, but costly, equipment such as reactive-ion etchers, contact aligners, or UV steppers. Instead, microfabrication was performed within a fume hood using routine laboratory equipment such as table top stands, ovens, and hand-machined plastic carriers made to fit bench top mechanical dimensions. This large benefit eases the adaptability of the bridged $\mu$ Lane to laboratories that are not microfluidics-based for use with a variety of samples and applications that range from examining particle distributions in environmental [63] to the study of cell [64] and bacterial chemotaxis [65]. 
In summary, we have developed the bridged $\mu$ Lane system to achieve autonomous and defined chemical transport within microenvironments using inexpensive fabrication and straightforward operation protocols. The bridged $\mu$ Lane system is a novel and user-friendly platform with which to generate and examine the effects of reagent concentration gradients in a multitude of scientific and engineering studies.

\section{Acknowledgment}

This work was supported by the National Institutes of Health (Grant No. GM071702), the National Science Foundation (Grant No. CBET0428573), and PSC-CUNY.

\section{References}

[1] Atencia, J., and Beebe, D. J., 2005, "Controlled Microfluidic Interfaces," Nature (London), 437(7059), pp. 648-655.

[2] Folch, A., 2007, "BioMEMS and Cellular Biology: Perspectives and Applications," J. Vis. Exp., (8), p. 300.

[3] Keenan, T. M., and Folch, A., 2007, "Biomolecular Gradients in Cell Culture Systems," Lab Chip, 8(1), pp. 34-57.

[4] Mosadegh, B., Huang, C., Park, J. W., Shin, H. S., Chung, B. G., Hwang, S K., Lee, K. H., Kim, H. J., Brody, J., and Jeon, N. L., 2007, "Generation of Stable Complex Gradients Across Two-Dimensional Surfaces and ThreeDimensional Gels," Langmuir, 23(22), pp. 10910-10912.

[5] Paguirigan, A. L., and Beebe, D. J., 2008, "Microfluidics Meet Cell Biology: Bridging the Gap by Validation and Application of Microscale Techniques for Cell Biological Assays," BioEssays, 30(9), pp. 811-821.

[6] Whitesides, G. M., 2006, "The Origins and the Future of Microfluidics," Nature (London), 442(7101), pp. 368-373.

[7] Kamotani, Y., Huh, D., Futai, N., and Takayama, S., 2007, "At the Interface: Advanced Microfluidic Assays for Study of Cell Function," BioMEMS and Biomedical Nanotechnology, Vol. 1, Springer Publishing, New York, pp. 5578 .

[8] McDonald, J., Duffy, D., Anderson, J., Chiu, D. T., Wu, H., Schueller, O., and Whitesides, G. M., 2000, "Fabrication of Microfluidic Systems in Poly(dimethylsiloxane)," Electrophoresis, 21(1), pp. 27-40.

[9] Weibel, D. B., and Whitesides, G. M., 2006, "Applications of Microfluidics in Chemical Biology," Curr. Opin. Chem. Biol., 10(6), pp. 584-591.

[10] Motoo, K., Toda, N., Arai, F., Fukuda, T., Sekiyama, K., and Nakajima, M., 2008, "Generation of Concentration Gradient From a Wave-Like Pattern by High Frequency Vibration of Liquid-Liquid Interface," Biomed. Microdevices, 10(3), pp. 329-335.

[11] Tanaka, Y., Morishima, K., Shimizu, T., Kikuchi, A., Yamato, M., Okano, T., and Kitamori, T., 2006, "Demonstration of a PDMS-Based Bio-Microactuator Using Cultured Cardiomyocytes to Drive Polymer Micropillars," Lab Chip, 6(2), pp. 230-235

[12] Yang, J., Yang, J., Yin, Z. Q., Svir, I., Xu, J., Luo, H. Y., Wang, M., Cao, Y., Hu, N., Liao, Y. J., and Zheng, X. L., 2009, "Microfluidic Pool Structure for Cell Docking and Rapid Mixing," Anal. Chim. Acta, 634(1), pp. 61-67.

[13] Ahmed, T., Shimizu, T. S., and Stocker, R., 2010, "Bacterial Chemotaxis in Linear and Nonlinear Steady Microfluidic Gradients," Nano Lett., 10(9), pp. 3379-3385.

[14] Balagadde, F. K., You, L., Hansen, C. L., Arnold, F. H., and Quake, S. R., 2005, "Long-Term Monitoring of Bacteria Undergoing Programmed Population Control in a Microchemostat," Science, 309(5731), pp. 137-140.

[15] Chung, S., Sudo, R., Mack, P. J., Wan, C. R., Vickerman, V., and Kamm, R. D., 2009, "Cell Migration Into Scaffolds Under Co-Culture Conditions in a Microfluidic Platform," Lab Chip, 9(2), pp. 269-275.

[16] Crowe, C. T., Elger, D. F., and Roberson, J. A., 2001, Engineering Fluid Mechanics, 7th ed., Wiley, New York.

[17] Dertinger, S., Chiu, D. T., Jeon, N. L., and Whitesides, G. M., 2001, "Generation of Gradients Having Complex Shapes Using Microfluidic Networks," Anal. Chem., 73, pp. 1240-1246.

[18] Jeon, N. L., Baskararn, H., Dertinger, S., Whitesides, G. M., Water, L. V., and Toner, M., 2002, "Neutrophil Chemotaxis in Linear and Complex Gradients of Interleukin-8 Formed in a Microfabricated Device," Nat. Biotechnol., 20, pp 826-830.

[19] Tourovskaia, A., Figueroa-Masot, X., and Folch, A., 2005, "Differentiationon-a-Chip: A Microfluidic Platform for Long-Term Cell Culture Studies,” Lab Chip, 5(1), pp. 14-19.

[20] Zaman, M. H., Trapani, L. M., Sieminski, A. L., Mackellar, D., Gong, H., Kamm, R. D., Wells, A., Lauffenburger, D. A., and Matsudaira, P., 2006, "Migration of Tumor Cells in 3D Matrices is Governed by Matrix Stiffness Along With Cell-Matrix Adhesion and Proteolysis," Proc. Natl. Acad. Sci. U.S.A., 103(29), pp. 10889-10894.

[21] Zhao, B., Moore, J. S., and Beebe, D. J., 2001, "Surface-Directed Liquid Flow Inside Microchannels," Science, 291(5506), pp. 1023-1026.

[22] Haibo, X., Yi, Z., Xin, F., Huayong, Y., and Hongyang, D., 2008, Study on Characteristics of Interface Between Multiple Laminar Streams and Application for Secondary Etching Inside Microchannels, Mechtroinc and Embedded Systems and Applications, MESA 2008, IEEE/ASME International Conference, pp. 243-248.
[23] Yamaguchi, Y., Takagi, F., Watari, T., Yamashita, K., Nakamura, H., Shimizu, H., and Maeda, H., 2004, "Interface Configuration of the Two Layered Laminar Flow in a Curved Microchannel," Chem. Eng. J., 101, pp. 367-372.

[24] Jeon, N. L., Dertinger, S., Chiu, D. T., Choi, I. S., Stroock, A. D., and Whitesides, G. M., 2000, "Generation of Solution and Surface Gradients Using Microfluidic Systems," Langmuir, 16, pp. 8311-8316.

[25] Du, Y., Shim, J., Vidula, M., Hancock, M. J., Lo, E., Chung, B. G., Borenstein, J. T., Khabiry, M., Cropek, D. M., and Khademhosseini, A., 2009, "Rapid Generation of Spatially and Temporally Controllable Long-Range Concentration Gradients in a Microfluidic Device," Lab Chip, 9(6), pp. 761-767.

[26] Beebe, D. J., Mensing, G. A., and Walker, G. M., 2002, "Physics and Applications of Microfluidics in Biology," Annu. Rev. Biomed. Eng., 4, pp. 261286.

[27] Izquierdo, S., Valdés, J. R., Martínez, M., Accolti, M., Woudberg, S., Asinari, P., Miana, M., and Du Plessis, J. P., 2010, "Porous-Layer Model for Laminar Liquid Flow in Rough Microchannels," Microfluid. Nanofluid., pp. 1-13.

[28] Bernard, A., Renault, J. P., Michel, B., Bosshard, H. R., and Delamarche, E., 2000, "Microcontact Printing of Proteins," Adv. Mater., 12(14), pp. 1067 1070.

[29] Delamarche, E., Bernard, A., Schmid, H., Michel, B., and Biebuyck, H., 1997, "Patterned Delivery of Immunoglobulins to Surfaces Using Microfluidic Networks," Science, 276(5313), pp. 779-781.

[30] Frevert, C. W., Boggy, G., Keenan, T. M., and Folch, A., 2006, "Measurement of Cell Migration in Response to an Evolving Radial Chemokine Gradient Triggered by a Microvalve," Lab Chip, 6(7), pp. 849-856.

[31] Goulpeau, J., Lonetti, B., Trouchet, D., Ajdari, A., and Tabeling, P., 2007, "Building up Longitudinal Concentration Gradients in Shallow Microchannels," Lab Chip, 7(9), pp. 1154-1161.

[32] Irimia, D., Geba, D. A., and Toner, M., 2006, "Universal Microfluidic Gradient Generator," Anal. Chem., 78(10), pp. 3472-3477.

[33] Kamholz, A. E., Weigl, B. H., Finlayson, B. A., and Yager, P., 1999, "Quantitative Analysis of Molecular Interaction in a Microfluidic Channel: The T-Sensor," Anal. Chem., 71(23), pp. 5340-5347.

[34] Tan, D. C., Yung, L. Y., and Roy, P., 2010, "Controlled Microscale Diffusion Gradients in Quiescent Extracellular Fluid," Biomed. Microdevices, 12(3), pp. 523-532.

[35] Abhyankar, V. V., Lokuta, M. A., Huttenlocher, A, and Beebe, D. J., 2006, "Characterization of a Membrane-Based Gradient Generator for Use in CellSignaling Studies," Lab Chip, 6(3), pp. 389-393.

[36] Fosser, K., and Nuzzo, R., 2003, "Fabrication of Patterned Multicomponent Protein Gradients and Gradient Arrays Using Microfluidic Depletion," Anal. Chem., 75, pp. 5775-5782.

[37] Kobel, S., and Lutolf, M., 2010, "High-Throughput Methods to Define Complex Stem Cell Niches," BioTechniques, 48(4), pp. ix-xxii.

[38] Millet, L. J., Stewart, M. E., Nuzzo, R. G., and Gillette, M. U., 2010, “Guiding Neuron Development With Planar Surface Gradients of Substrate Cues Deposited Using Microfluidic Devices," Lab Chip, 10(12), pp. 1525-1535.

[39] Wu, H., Huang, B., and Zare, R. N., 2006, "Generation of Complex, Static Solution Gradients in Microfluidic Channels," J. Am. Chem. Soc., 128(13), pp. 4194-4195.

[40] Li, C. W., Chen, R., and Yang, M., 2007, "Generation of Linear and NonLinear Concentration Gradients Along Microfluidic Channel by Microtunnel Controlled Stepwise Addition of Sample Solution," Lab Chip, 7(10), pp. 1371-1373.

[41] Paliwal, S., Iglesias, P. A Campbell, K., Hilioti, Z., Groisman, A., and Levchenko, A., 2007, "MAPK-Mediated Bimodal Gene Expression and Adaptive Gradient Sensing in Yeast," Nature (London), 446(7131), pp. 46-51.

[42] Kamholz, A. E., and Yager, P., 2001, "Theoretical Analysis of Molecular Diffusion in Pressure-Driven Laminar Flow in Microfluidic Channels," Biophys. J., 80(1), pp. 155-160.

[43] Breckenridge, M. T., Egelhoff, T. T., and Baskaran, H., 2010, "A Microfluidic Imaging Chamber for the Direct Observation of Chemotactic Transmigration,' Biomed. Microdevices, 12(3), pp. 543-553.

[44] Lee, J., Hu, Y., and Li, D., 2005, "Electrokinetic Concentration Gradient Generation Using a Converging-Diverging Microchannel," Anal. Chim. Acta, 543(1-2), pp. 99-108.

[45] Velve-Casquillas, G., Berrea, M. L., Piela, M., and Tran, P. T., 2010, "Microfluidic Tools for Cell Biological Research," Nanotoday, 5(1), pp. 28-47.

[46] Saadi, W., Rhee, S. W., Lin, F., Vahidi, B., Chung, B. G., and Jeon, N. L., 2007, "Generation of Stable Concentration Gradients in 2D and 3D Environments Using a Microfluidic Ladder Chamber," Biomed. Microdevices, 9(5), pp. 627-635.

[47] Kanegasaki, S., Nomura, Y., Nitta, N., Akiyama, S., Tamatani, T., Goshoh, Y, Yoshida, T., Sato, T., and Kikuchi, Y., 2003, “A Novel Optical Assay System for the Quantitative Measurement of Chemotaxis," J. Immunol. Methods, 282(1-2), pp. 1-11.

[48] Lin, F., 2004, "Generation of Dynamic Temporal and Spatial Concentration Gradients Using Microfluidic Devices," Lab on a Chip, 4(3), pp. 164-167.

[49] Adler, M., Polinkovsky, M., Gutierrez, E., and Groisman, A., 2010, "Generation of Oxygen Gradients With Arbitrary Shapes in a Microfluidic Device," Lab Chip, 10(3), pp. 388-391.

[50] Park, J. Y., Kim, S. K., Woo, D. H., Lee, E. J., Kim, J. H., and Lee, S. H., 2009, "Differentiation of Neural Progenitor Cells in a Microfluidic ChipGenerated Cytokine Gradient," Stem Cells, 27(11), pp. 2646-2654.

[51] Lee, I., Chan, K., and Phillips, D. L., 1998, "Growth of Electrodeposited Platinum Nanocrystals Studied by Atomic Force Microscopy," Appl. Surf. Sci., 136(4), pp. 321-330. 
[52] McKnight, T. E., Melechko, A. V., Austin, D. W., Sims, T., Guillorn, M. A., and Simpson, M. L., 2004, "Microarrays of Vertically-Aligned Carbon Nanofiber Electrodes in an Open Fluidic Channel," J. Phys. Chem. B, 108, pp. 7115-7125

[53] Vazquez, M., McKinley, G., Mitnik, L., Desmarais, S., Matsudaira, P., and Ehrlich, D., 2002, "Electrophoretic Injection Within Microdevices," Anal. Chem., 74(9), pp. 1952-1961.

[54] Vazquez, M., Schmalzing, D., Matsudaira, P., Ehrlich, D., and McKinley, G., 2001, "Shear-Induced Degradation of Linear Polyacrylamide Solutions During Pre-Electrophoretic Loading," Anal. Chem., 73(13), pp. 3035-3044.

[55] Holden, M. A., Kumar, S., Castellana, E. T., Beskok, A., and Cremer, P. S., 2003, "Generating Fixed Concentration Arrays in a Microfluidic Device," Sens. Actuators B, 92, pp. 199-207.

[56] Bird, R. B., Stewart, W. E., and Lightfoot, E. N., 2002, Transport Phenomena, 2nd ed., Wiley, New York.

[57] Feng, J. J., Wang, A. J., Fan, J., Xu, J. J., and Chen, H. Y., 2010, "Hydrophilic Biopolymer Grafted on Poly(dimethylsiloxane) Surface for Microchip Electrophoresis," Anal. Chim. Acta, 658(1), pp. 75-80.

[58] Gennerich, A., and Schild, D., 2002, "Anisotropic Diffusion in Mitral Cell
Dendrites Revealed by Fluorescence Correction Spectroscopy,” Biophys. J., 83, pp. 510-522.

[59] Haller, M. F., and Saltzman, W. M., 1998, "Localized Delivery of Proteins in the Brain: Can Transport Be Customized?," Pharm. Res., 15(3), pp. 377-385.

[60] Hiemenz, P. C., and Rajagopalan, R., 1997, Principles of Colloid and Surface Chemistry, 3rd ed., Marcel Dekker, New York.

[61] Ioan, C. E., Aberle, T., and Burchard, W., 2000, "Structure Properties of Dextran. 2. Dilute Solution," Macromolecules, 33(15), pp. 5730-5739.

[62] Saltzman, W. M., Radomsky, M. L., Whaley, K. J., and Cone, R. A., 1994, "Antibody Diffusion in Human Cervical Mucus," Biophys. J., 66(2), pp. 508515.

[63] Yamada, M., and Seki, M., 2006, "Microfluidic Particle Sorter Employing Flow Splitting and Recombining," Anal. Chem., 78(4), pp. 1357-1362.

[64] Kim, D., Lokuta, M. A., Huttenlocher, A., and Beebe, D. J., 2009, "Selective and Tunable Gradient Device for Cell Culture and Chemotaxis Study," Lab Chip, 9(12), pp. 1797-1800.

[65] Englert, D. L., Manson, M. D., and Jayaraman, A., 2010, "Investigation of Bacterial Chemotaxis in Flow-Based Microfluidic Devices," Nat. Protoc., 5(5), pp. 864-872. 